\title{
Multiantenna Analog Network Coding for Multihop Wireless Networks
}

\author{
Ramesh Annavajjala, Amine Maaref, and Jinyun Zhang \\ Mitsubishi Electric Research Laboratories (MERL), 201 Broadway Cambridge, MA 02139, USA \\ Correspondence should be addressed to Ramesh Annavajjala, ramesh.annavajjala@gmail.com
}

Received 16 July 2009; Accepted 26 October 2009

Academic Editor: Guoqing Li

Copyright (๑) 2010 Ramesh Annavajjala et al. This is an open access article distributed under the Creative Commons Attribution License, which permits unrestricted use, distribution, and reproduction in any medium, provided the original work is properly cited.

\begin{abstract}
This paper proposes a two-phase minimum mean-square-error bidirectional amplify-and forward (MMSE-BAF) relaying protocol to allow two sources exchange independent messages via a relay node equipped with multiple antennas. MMSE-BAF performs a joint linear MMSE filtering of the received signal after the multiple access phase before amplifying and forwarding the filtered signal using a single transmit antenna, possibly through a specific antenna selection procedure, during the broadcast phase. The proposed protocol extends upon the so-called analog network coding schemes in the literature in that it inherently exploits the multiple antennas at the relay station to reduce the noise enhancement typical of an AF protocol, and can also compensate for link imbalances between the relay and the sources and is agnostic to sources' modulation and coding schemes. We derive the instantaneous signal-to-noise ratio expressions for the received signal by the sources in the downlink and provide extensive linklevel simulations for the MMSE-BAF protocol subject to both frequency flat and selective fading. Furthermore, we pinpoint the modifications to be incorporated into the IEEE 802.16e orthogonal-frequency-division multiple access (OFDMA) cellular standard (mobile WiMax) to enable support of multiantenna bidirectional communications and show that MMSE-BAF is a viable solution within that framework.
\end{abstract}

\section{Introduction}

Half-duplex bidirectional relay systems in which two nodes $S_{1}$ and $S_{2}$ wish to exchange independent messages via a third node $R$, termed relay, give rise to some interesting challenges from a cooperative communications and informationtheoretic points of view. This is especially true when the relay node $R$ is equipped with multiple antennas. Such two-way relay channels have many applications in ad hoc and cellular networks in which all mobile-to-mobile communications have to pass through a common base station. Since fullduplex operation is of little practical interest given current state-of-the-art technology, our focus is on half-duplex nodes, where each active node can either transmit or receive an information message at a given point in time. In particular, without loss of generality, we are interested in the communications part of the problem in a cellular context where two mobile stations wish to exchange data simultaneously via a common base station.
The traditional baseline approach for bidirectional communications in half-duplex mode between two sources $S_{1}$ and $S_{2}$ via a relay station $R$ consists of a 4-phase protocol with a completion time of 4-time-slots (TSs) whereby $S_{1}$ and $S_{2}$ send $N$-bit packets $\mathbf{b}_{1}$ and $\mathbf{b}_{2} \in\{0,1\}^{N}$ to $R$ during TS 1 and TS 2 , respectively; $R$ decodes the received packets and then sends $\mathbf{b}_{2}$ to $S_{1}$ and $\mathbf{b}_{1}$ to $S_{2}$ during TS 3 and TS 4 , respectively. The gist of the 4-phase protocol is to avoid interference by preventing simultaneous transmissions from the sources to the relay and vice versa. However, it was shown in [1] that a three-phase protocol exploiting the so-called network coding concept by decoding packets $\mathbf{b}_{1}$ and $\mathbf{b}_{2}$ at the relay and then broadcasting a single packet $\mathbf{b}_{1} \oplus \mathbf{b}_{2}$, where $\oplus$ denotes the bitwise exclusive-or (XOR) operation, is actually more attractive in terms of achievable throughput, since the desired packet at $S_{1}$ can be decoded using another XOR operation (and similarly at $S_{1}$ ). Better still, a recent concept introduced in [2] and termed analog network coding (ANC) combines the first two phases of the conventional baseline protocol into a single 


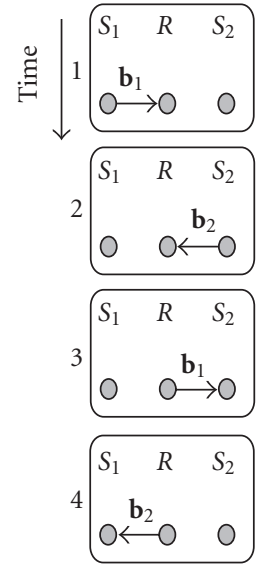

(a)
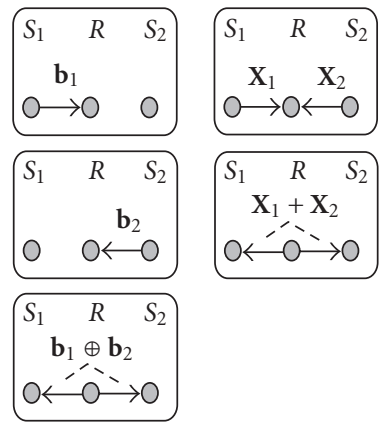

(b)

(c)
Figure 1: Bidirectional communication protocols: (a) 4-phase conventional protocol, (b) 3-phase protocol with decode-andforward network coding, and (c) 2-phase BAF protocol with amplify-and-forward relaying.

multiple access (MA) phase with simultaneous transmissions from the sources to the relay; the received multiple access signal at the relay is then amplified and broadcast to $S_{1}$ and $S_{2}$, thereby yielding the so-called two-phase bidirectional amplify-and-forward (BAF) protocol. A similar concept to ANC, using estimate-and-forward relaying as opposed to AF relaying, has been proposed in [3] under the terminology of physical-layer network coding (PNC). A schematic diagram illustrating the aforementioned bidirectional protocols is illustrated in Figure 1.

Information-theoretic aspects such as bounds on the achievable throughput and the capacity region of the bidirectional relay channel have been investigated in [46]. A Markov-chain-based performance analysis for several variants of the BAF protocol was carried out in [7-9]. Linear beamforming filter designs for bidirectional communications with multiantenna relay stations are proposed in $[10$ 12]. In this paper, we propose a simple two-phase minimummean-square-error- (MMSE-) BAF protocol which operates by filtering the received signal at the relay station after the MA phase using a specially designed joint linear MMSE filter before amplifying and forwarding the filtered signal during the broadcast phase. Whereas optimal relay beamforming structure for bidirectional multiantenna relay channels is sought in [12], our MMSE-BAF protocol is a simple lowcomplexity driven approach for bidirectional multiantenna relay channels which exploits linear signal processing on the uplink (from $S_{1}$ and $S_{2}$ to $R$ ) and transmit antenna selection (TAS) on the downlink (from $R$ to $S_{1}$ and $S_{2}$ ). Besides, MMSE-BAF is a two-phase bidirectional relaying protocol, whereas the multiantenna relaying protocol put forward in [10] is a three-phase protocol which necessitates decoding and reencoding of the received signals at the RS prior to the broadcast phase. Finally, MMSE-BAF differs from the so-called spatial division duplex (SDD) bidirectional relaying scheme proposed in [11] in that it allows to bias the beamforming weights in favor of one of the two source nodes in as much as required to compensate for potential imbalance of the relay-to-source link channel gains or other parameters such as dissimilar signal constellations employed at $S_{1}$ and $S_{2}$.

The remainder of this paper is structured as follows. Section 2 presents the system model for MMSE-BAF relaying. In Section 3, we describe the proposed MMSE-BAF protocol and derive the signal-to-noise ratio (SNR) expressions upon which our extensive simulation results provided in Section 4 are based. Finally, concluding remarks are drawn in Section 5.

\section{System Setup and Signal Model}

The following set of notations is employed throughout this paper: Boldface upper- and lower-case symbols are used to denote matrices and column vectors, respectively. $\mathbf{I}_{m}$ denotes the identity matrix of order $m$. Moreover, $(\cdot)^{*},(\cdot)^{T},(\cdot)^{\mathscr{H}}$, and $\mathrm{E}[\cdot]$ stand for conjugate, transpose, transpose-conjugate, and expectation operators, respectively.

Without loss of generality, and although our proposal and framework are applicable to any type of two-hop bidirectional relay setting, we focus our attention on cellular systems. For that purpose, we consider an infrastructurebased wireless communications system consisting of two mobile stations (MSs), $\mathrm{MS}_{1}$ and $\mathrm{MS}_{2}$, and one base station (BS). A block diagram of the system under consideration is depicted in Figure 2. Both MSs as well as the BS are equipped with multiple antennas for reception with the aim of canceling out potential other cell/user interference but are restricted to transmit through a single transmit antenna. This assumption is dictated by the need to reduce the transmitpower requirements for user terminals and to lower the complexity and cost of a transmission chain at the base station, generally higher than that of a reception chain, especially when accounting for high-cost radio-frequency amplifiers involved in the transmission chain. This is, for instance, the case in current cellular standards such as IEEE 802.16e [13]. Note that our proposed scheme works equally well with both time-division duplex (TDD) as well as frequency-division duplex (FDD) modes of operation. Without loss of generality and for the sake of notational brevity, we focus on the FDD mode in the following analysis. Performance results for both TDD and FDD will be presented in Section 4.

Complex baseband transmission is assumed throughout the paper. Let $M_{\mathrm{bs}}$ denote the number of receive antennas at the BS, and let $\mathbf{h}_{1}[n]=\left[h_{1}^{1}[n], h_{1}^{2}[n], \ldots, h_{1}^{M_{\mathrm{bs}}}[n]\right]^{T}$ and $\mathbf{h}_{2}[n]=\left[h_{2}^{1}[n], h_{2}^{2}[n], \ldots, h_{2}^{M_{\mathrm{bs}}}[n]\right]^{T}$ denote the $M_{\mathrm{bs}} \times 1$ uplink channels from $\mathrm{MS}_{1}$ and $\mathrm{MS}_{2}$ to the BS, respectively, where $n$ is the discrete-time index. The corresponding time-varying channel vector elements $\left\{h_{i}^{j}[n]\right\}_{i=1,2 ; j=1, \ldots, M_{\mathrm{bs}}}$ are realizations of a zero-mean unit-variance Gaussian wide-sense stationary process. Assuming $M_{s s}$ antennas at each of $\mathrm{MS}_{1}$ and $\mathrm{MS}_{2}$ for downlink reception, we define $\mathbf{g}_{1}[n]=\left[g_{1}^{1}[n], g_{1}^{2}[n], \ldots, g_{1}^{M_{s s}}[n]\right]^{T}$ and $\mathbf{g}_{2}[n]=$ $\left[g_{2}^{1}[n], g_{2}^{2}[n], \ldots, g_{2}^{M_{s s}}[n]\right]^{T}$ as the downlink channels from 


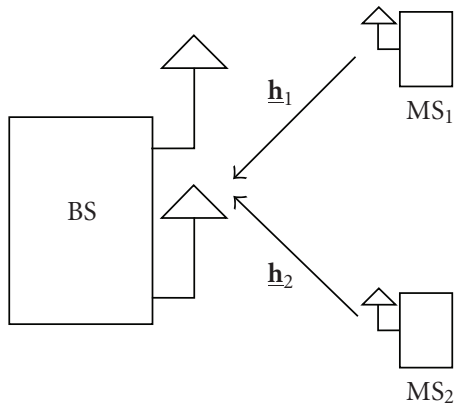

(a) Uplink multiple access phase

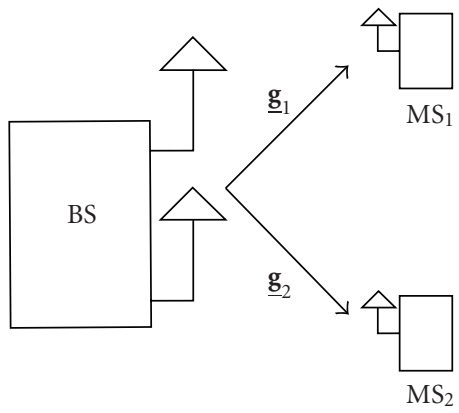

(b) Dowlink broadcast phase

FIGURE 2: Block diagram of the system setup.

BS to $\mathrm{MS}_{1}$ and $\mathrm{MS}_{2}$, respectively. For the special case of $M_{\mathrm{bs}}=M_{s s}=1$, TDD assumption allows us to set $\mathbf{g}_{1}[n]=$ $\mathbf{h}_{1}^{T}[n]$ and $\mathbf{g}_{2}=\mathbf{h}_{2}^{T}[n]$. Let $x_{1}[n]$ be the signal transmitted from $\mathrm{MS}_{1}$ and intended for $\mathrm{MS}_{2}$ and let $x_{2}[n]$ be the signal transmitted from $\mathrm{MS}_{2}$ and intended for $\mathrm{MS}_{1}$ at time $n$. Both $x_{1}[n]$ and $x_{2}[n]$ are drawn from two possibly different complex signal constellations with average energies $\sigma_{1}^{2}=$ $\mathrm{E}\left[\left|x_{1}[n]\right|^{2}\right]$ and $\sigma_{2}^{2}=\mathrm{E}\left[\left|x_{2}[n]\right|^{2}\right]$, respectively. Prior to any signal processing at the BS, the $M_{\mathrm{bs}} \times 1$-received signal at the end of the MA phase is given by

$$
\mathbf{y}[n]=\mathbf{h}_{1}[n] x_{1}[n]+\mathbf{h}_{2}[n] x_{2}[n]+\mathbf{n}[n],
$$

where $\mathbf{n}[n]$ is an $M_{\mathrm{bs}} \times 1$ additive white Gaussian noise vector at the BS which is modeled as a zero-mean circularly symmetric Gaussian random vector with covariance matrix $\mathrm{E}\left[\mathbf{n}[n] \mathbf{n}[n]^{\mathscr{H}}\right]=\sigma_{N}^{2} \mathbf{I}_{M_{\mathrm{bs}}}$. For notational simplicity, the discrete-time index $n$ is henceforth omitted.

\section{MMSE-BAF: Description and Analysis}

3.1. Protocol Description. Figure 3 illustrates the physicallayer frame structure for enabling the MMSE-BAF protocol for the uplink MA and the downlink broadcast phases. A frame consists of packets originating from the linklayer whose size depends on the chosen MCS so that each downlink or uplink frame contains a fixed number of symbols. As can be seen in the left-hand side of Figure 3, the uplink frame structure is composed of two parts, one for pilot symbols which are chosen to be orthogonal for $\mathrm{MS}_{1}$ and $\mathrm{MS}_{2}$. Orthogonality of the pilot symbols can be maintained in the time, frequency, or two-dimensional time-frequency grid. The second part is for data symbols. The orthogonal pilot symbols are used to estimate the channels $\mathbf{h}_{1}$ and $\mathbf{h}_{2}$ corresponding to $\mathrm{MS}_{1}$ and $\mathrm{MS}_{2}$. A beamforming weight vector $\mathbf{w}_{\text {opt }}$ is then computed at the BS based on a joint MMSE criterion to be specified shortly. The BS then estimates an amplification factor $\beta$ subject to an average power constraint. Likewise, the downlink frame structure contains pilot and data parts. Additionally, it contains a control part consisting of quantized versions of the amplification factor $\beta$ (a positive scalar value) and two complex scalars values $v_{1}:=\mathbf{w}_{\mathrm{opt}}^{\mathcal{H}} \mathbf{h}_{1}$ and $v_{2}:=\mathbf{w}_{\mathrm{opt}}^{\mathcal{H}} \mathbf{h}_{2}$. The downlink data symbols consist of the beamformed amplified-and-forwarded symbols received on the uplink frame in FDD mode.

According to the MMSE-BAF protocol, the BS performs the following set of operations.

(1) Jointly minimize the MSE between the received signal $\mathbf{y}[n]$ and the transmitted signals $x_{1}[n]$ and $x_{2}[n]$, thus performing a joint linear-MMSE filtering of the received signal, using the following metric:

$$
\begin{aligned}
\mathbf{w}_{\mathrm{opt}}=\underset{\mathbf{w} \in \mathbb{C}^{M_{\mathrm{bs}}}}{\operatorname{argmin}}\{ & \delta_{1} \mathrm{E}\left[\left|x_{1}-\mathbf{w}^{\mathcal{H}} \mathbf{y}\right|^{2} \mid \mathbf{h}_{1}, \mathbf{h}_{2}\right] \\
& \left.+\delta_{2} \mathrm{E}\left[\left|x_{2}-\mathbf{w}^{\mathcal{H}} \mathbf{y}\right|^{2} \mid \mathbf{h}_{1}, \mathbf{h}_{2}\right]\right\},
\end{aligned}
$$

where $\mathbb{C}$ is the field of complex numbers and $\delta_{1} \geq 0$, $\delta_{2} \geq 0, \delta_{1}+\delta_{2}=1$, are the two design constants that control the relative weight assigned to the signals of $\mathrm{MS}_{1}$ and $\mathrm{MS}_{2}$. The minimization problem in (2) is a modified Wiener filtering problem whose solution can be easily found using the orthogonality principal in linear mean square estimation and is given by

$$
\mathbf{w}_{\mathrm{opt}}=\left(\sigma_{1}^{2} \mathbf{h}_{1} \mathbf{h}_{1}^{\mathcal{H}}+\sigma_{2}^{2} \mathbf{h}_{2} \mathbf{h}_{2}^{\mathcal{H}}+\sigma_{N}^{2} \mathbf{I}_{M_{\mathrm{bs}}}\right)^{-1}\left(\delta_{1} \sigma_{1}^{2} \mathbf{h}_{1}+\delta_{2} \sigma_{2}^{2} \mathbf{h}_{2}\right) .
$$

This minimization requires an estimation of both mobile stations' vector-valued channels $\mathbf{h}_{1}$ and $\mathbf{h}_{2}$.

(2) Amplify the linear MMSE-filter output to maintain a constant average transmit power $P_{\mathrm{T}}$ which leads to computing the amplification gain factor:

$$
\begin{aligned}
\beta & =\sqrt{\frac{P_{\mathrm{T}}}{\mathrm{E}\left[\left|\mathbf{w}^{\mathcal{H}} \mathbf{y}\right|^{2} \mid \mathbf{h}_{1}, \mathbf{h}_{2}\right]}} \\
& =\sqrt{\frac{P_{\mathrm{T}}}{\sigma_{1}^{2}\left|\mathbf{w}_{\mathrm{opt}}^{\mathcal{H}} \mathbf{h}_{1}\right|^{2}+\sigma_{2}^{2}\left|\mathbf{w}_{\mathrm{opt}}^{\mathcal{H}} \mathbf{h}_{2}\right|^{2}+\sigma_{N}^{2}|| \mathbf{w}_{\mathrm{opt}} \|^{2}}} .
\end{aligned}
$$

(3) Transmit the amplified signal back to the MSs on one of the antennas using an appropriate downlink transmit antenna-selection (TAS) algorithm, based on the uplink channel. One approach inherent to the MMSE-BAF protocol is to select the antenna that has the largest beamformer weight. We note that, owing to the channel reciprocity, TAS using the largest beamformer weight is applicable for TDD only. 


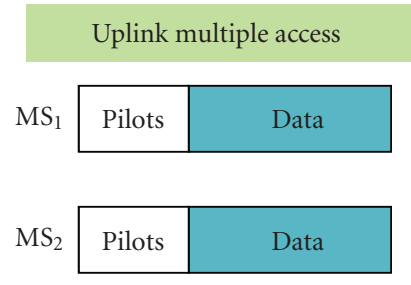

(a)

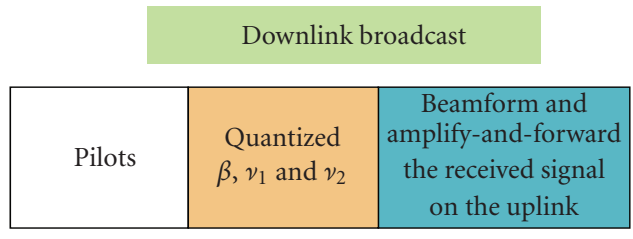

(b)

Figure 3: Block diagram of the system setup.

3.2. Performance Analysis. Define $z:=\mathbf{w}_{\mathrm{opt}}^{\mathscr{H}} \mathbf{y}$, where $\mathbf{y}$ is the uplink received signal (1), as the output of the MMSE filtering operation at the $\mathrm{BS}$. The AF transmitted signal on the downlink is

$$
x_{r}=\beta z=\beta \mathbf{w}_{\mathrm{opt}}^{\mathcal{H}} \mathbf{y} .
$$

The received signal on the downlink for $\mathrm{MS}_{1}$ and $\mathrm{MS}_{2}$ is therefore given by

$$
\begin{aligned}
& \mathbf{y}_{1}=\mathbf{g}_{1} x_{r}+\mathbf{n}_{1}, \\
& \mathbf{y}_{2}=\mathbf{g}_{2} x_{r}+\mathbf{n}_{2},
\end{aligned}
$$

where $\mathbf{n}_{1}$ and $\mathbf{n}_{2}$ are the zero-mean AWGN at MS ${ }_{1}$ and $\mathrm{MS}_{2}$, respectively, with covariance matrix $\sigma_{N}^{2} \mathbf{I}_{M_{s s}}$. Without loss of generality, let us focus on the signal received by $\mathrm{MS}_{1}$ (6). A similar signal processing is required at $\mathrm{MS}_{2}$. Incorporating (1) and (5) into (6) yields

$$
\mathbf{y}_{1}=\mathbf{g}_{1} \beta \mathbf{w}_{\mathrm{opt}}^{\mathcal{H}}\left(\mathbf{h}_{1} x_{1}+\mathbf{h}_{2} x_{2}+\mathbf{n}\right)+\mathbf{n}_{1} .
$$

Now, assuming that $\mathrm{MS}_{1}$ (i) is able to perfectly estimate its own downlink channel vector $\mathbf{g}_{1}$ owing to the downlink pilot symbols sent by the BS, (ii) knows its own transmitted signal $x_{1}$, and (iii) is able to extract the value of the amplification factor $\beta$ as well as the couple $\left(v_{1}, v_{2}\right)$, all of which are sent on the downlink control channel as explained in Section 3.1, then $\mathrm{MS}_{1}$ can subtract the so-called self-interference component of the received signal, that is, $\mathbf{g}_{1} \beta v_{1} x_{1}$, hence winding up with a processed received signal of the form

$$
\begin{aligned}
\mathbf{r}_{1} & =\mathbf{y}_{1}-\mathbf{g}_{1} \beta v_{1} x_{1} \\
& =\mathbf{y}_{1}-\mathbf{g}_{1} \beta \mathbf{w}_{\mathrm{opt}}^{\mathcal{H}} \mathbf{h}_{1} x_{1} \\
& =\mathbf{g}_{1} \beta \mathbf{w}_{\mathrm{opt}}^{\mathcal{H}} \mathbf{h}_{2} x_{2}+\underbrace{\mathbf{g}_{1} \beta \mathbf{w}_{\mathrm{opp}}^{\mathcal{H}} \mathbf{n}+\mathbf{n}_{1}}_{:=\tilde{\mathbf{n}}_{1}} .
\end{aligned}
$$

Note that $\widetilde{\mathbf{n}}_{1}$, defined in the previous equation, is a zeromean colored noise vector with a conditional covariance matrix given by

$$
\begin{aligned}
\boldsymbol{\Sigma}_{1} & :=\mathrm{E}\left[\tilde{\mathbf{n}}_{1} \tilde{\mathbf{n}}_{1}^{\mathcal{H}} \mid \mathbf{g}_{1}, \beta, \mathbf{w}_{\mathrm{opt}}\right] \\
& =\beta^{2} \mathbf{g}_{1} \mathbf{w}_{\mathrm{opt}}^{\mathscr{H}} \mathrm{E}\left[\mathbf{n} \mathbf{n}^{\mathcal{H}}\right] \mathbf{w}_{\mathrm{opt}} \mathbf{g}_{1}^{\mathcal{H}}+\mathrm{E}\left[\mathbf{n}_{1} \mathbf{n}_{1}^{\mathcal{H}}\right] \\
& =\sigma_{N}^{2}\left(\mathbf{I}_{M_{s s}}+\beta^{2} \mathbf{g}_{1} \mathbf{w}_{\mathrm{opt}}^{\mathcal{H}} \mathbf{w}_{\mathrm{opt}} \mathbf{g}_{1}^{\mathscr{H}}\right) .
\end{aligned}
$$

In the absence of knowledge of the conditional covariance matrix $\boldsymbol{\Sigma}_{1}$ at the receiver of $\mathrm{MS}_{1}$, an estimate $\hat{x}_{2}$ of $x_{2}$ can be obtained as follows:

$$
\hat{x}_{2}=\frac{\left(\mathbf{g}_{1} \beta v_{2}\right)^{\mathscr{H}}}{\left(\mathbf{g}_{1} \beta v_{2}\right)^{\mathscr{H}}\left(\mathbf{g}_{1} \beta v_{2}\right)} \mathbf{r}_{1},
$$

which after simplification becomes

$$
\hat{x}_{2}=x_{2}+\underbrace{\frac{1}{\beta v_{2}} \frac{\mathbf{g}_{1}^{\mathscr{H}} \widetilde{\mathbf{n}}_{1}}{\mathbf{g}_{1}^{\mathscr{H}} \mathbf{g}_{1}}}_{:=\check{n}_{2}} .
$$

The conditional variance of the newly defined scalar noise term $\check{n}_{2}$ can be expressed as

$$
\begin{aligned}
\Sigma_{1}^{\prime}=\mathrm{E}\left[\left|\check{n}_{2}\right|^{2}\right] & =\frac{1}{\beta^{2}\left|v_{2}\right|^{2}} \frac{\mathbf{g}_{1}^{\mathscr{H}} \mathrm{E}\left[\widetilde{\mathbf{n}}_{1}^{\mathcal{H}} \widetilde{\mathbf{n}}_{1}\right] \mathbf{g}_{1}}{\left(\mathbf{g}_{1}^{\mathcal{H}} \mathbf{g}_{1}\right)^{2}} \\
& =\frac{1}{\beta^{2}\left|v_{2}\right|^{2}} \frac{\mathbf{g}_{1}^{\mathcal{H}} \boldsymbol{\Sigma}_{1} \mathbf{g}_{1}}{\left(\mathbf{g}_{1}^{\mathcal{H}} \mathbf{g}_{1}\right)^{2}} .
\end{aligned}
$$

Now, we are in a position to determine the $\mathrm{SNR} \gamma_{2}$ at $\mathrm{MS}_{1}$ as

$$
\begin{aligned}
\gamma_{2} & =\frac{\mathrm{E}\left[\left|x_{2}\right|^{2}\right]}{\mathrm{E}\left[\left|\check{n}_{2}\right|^{2}\right]} \\
& =\frac{\sigma_{2}^{2} \beta^{2}\left|v_{2}\right|^{2}\left(\mathbf{g}_{1}^{\mathcal{H}} \mathbf{g}_{1}\right)^{2}}{\mathbf{g}_{1}^{\mathcal{H}} \Sigma_{1} \mathbf{g}_{1}} .
\end{aligned}
$$

Note that after incorporating (10) into (17) and further simplification, (17) can be expressed as follows:

$$
\gamma_{2}=\frac{\sigma_{2}^{2} \beta^{2}\left|v_{2}\right|^{2}}{\sigma_{N}^{2}} \times \frac{\left\|\mathbf{g}_{1}\right\|^{2}}{1+\beta^{2}\left\|\mathbf{w}_{\mathrm{opt}}\right\|^{2}\left\|\mathbf{g}_{1}\right\|^{2}} .
$$

Similarly, one can evaluate the SNR $\gamma_{1}$ for the signal $x_{1}$ received at $\mathrm{MS}_{2}$ which is found to be

$$
\gamma_{1}=\frac{\sigma_{1}^{2} \beta^{2}\left|v_{1}\right|^{2}\left(\mathbf{g}_{2}^{\mathscr{H}} \mathbf{g}_{2}\right)^{2}}{\mathbf{g}_{2}^{\mathscr{H}} \boldsymbol{\Sigma}_{2} \mathbf{g}_{2}},
$$

where $\boldsymbol{\Sigma}_{2}$ is a noise covariance matrix (analogous to $\boldsymbol{\Sigma}_{1}$ ) defined as

$$
\boldsymbol{\Sigma}_{2}:=\sigma_{N}^{2}\left(\mathbf{I}_{M_{\mathrm{ss}}}+\beta^{2} \mathbf{g}_{2} \mathbf{w}_{\mathrm{opt}}^{\mathscr{H}} \mathbf{w}_{\mathrm{opt}} \mathbf{g}_{2}^{\mathcal{H}}\right) .
$$


Again, upon incorporation of (20) into (19), the latter can be simplified to

$$
\gamma_{1}=\frac{\sigma_{1}^{2} \beta^{2}\left|v_{1}\right|^{2}}{\sigma_{N}^{2}} \times \frac{\left\|\mathbf{g}_{2}\right\|^{2}}{1+\beta^{2}\left\|\mathbf{w}_{\mathrm{opt}}\right\|^{2}\left\|\mathbf{g}_{2}\right\|^{2}} .
$$

It is worthwhile to mention that the above SNR expressions for $\gamma_{2}$ (18) and $\gamma_{1}$ (21) have been obtained without exploiting the colored nature of $\boldsymbol{\Sigma}_{1}$ and $\boldsymbol{\Sigma}_{2}$. Somewhat surprisingly, and as indicated in the appendix, even through whitening of the colored noise, the SNR expressions for $\gamma_{1}$ and $\gamma_{2}$ remain the same, which is not so bad in itself, as it means that the signal processing cost associated with the whitening operation can be completed eliminated.

\section{Simulation Results and Discussion}

In this section, we present some simulation results on the performance of the proposed MMSE-BAF protocol. First, Figure 4 shows the MSE performance of MMSE-BAF as a function of the relative loading of user-1 over user2, $\delta_{1}$, with $M_{\mathrm{bs}}=4$ antennas at the base-station. Two scenarios are considered: in Figure 4(a), both users average received SNRs at the base-station are set to $10 \mathrm{~dB}$, whereas in Figure 4(b) the average received SNR is set to $20 \mathrm{~dB}$ and $40 \mathrm{~dB}$ for user- 1 and user-2, respectively. The weighted average MSE immediately after the application of MMSE beamforming and the average MSE of user-1 and user-2 after MMSE beamforming followed by AF are obtained by drawing independent channel realizations over 100000 trials. From Figures 4(a) and 4(b), we observe that increasing $\delta_{1}$ minimizes the MSE of user- 1 at the expense of an increase in MSE for user-2, whereas an optimum $\delta_{1}$ exists that jointly minimizes the MSE of both users. Interestingly, from an implementation point of view, the range of $\delta_{1}$ is broad to arrive at this optimum overall MSE. Comparing Figures 4(a) and $4(\mathrm{~b})$, we notice that, due to unequal average received SNRs, the individual MSEs as well as the overall MSE are not symmetric functions of $\delta_{1}$. Thus, one should take into account the knowledge of the average uplink SNRs to arrive at an appropriate $\delta_{1}$ to maintain desired MSE levels for each of the two users.

In Figure 5, the empirical cumulative distribution function (CDF) of two-time-slots-based MMSE-BAF is compared against the four-time-slot-based baseline system. Here, the base-station and the mobile station receivers are each equipped with four receive antennas. The uplink average received SNRs, per antenna, of users 1 and 2 are set to $5 \mathrm{~dB}$ and $10 \mathrm{~dB}$, respectively, and the average received SNR per antenna at each mobile station is set to $5 \mathrm{~dB}$. We also assume $\delta_{1}=\delta_{2}$. Figure 5 shows that the two-time-slotsbased MMSE-BAF system outperforms the baseline system by an order of magnitude.

As argued earlier, the proposed MMSE-BAF protocol is equally attractive to both TDD and FDD systems. Figures 6 and 7 show uncoded symbol error rate (SER) performance of MMSE-BAF on block-fading TDD channels when the two users employ dissimilar modulation formats. In both

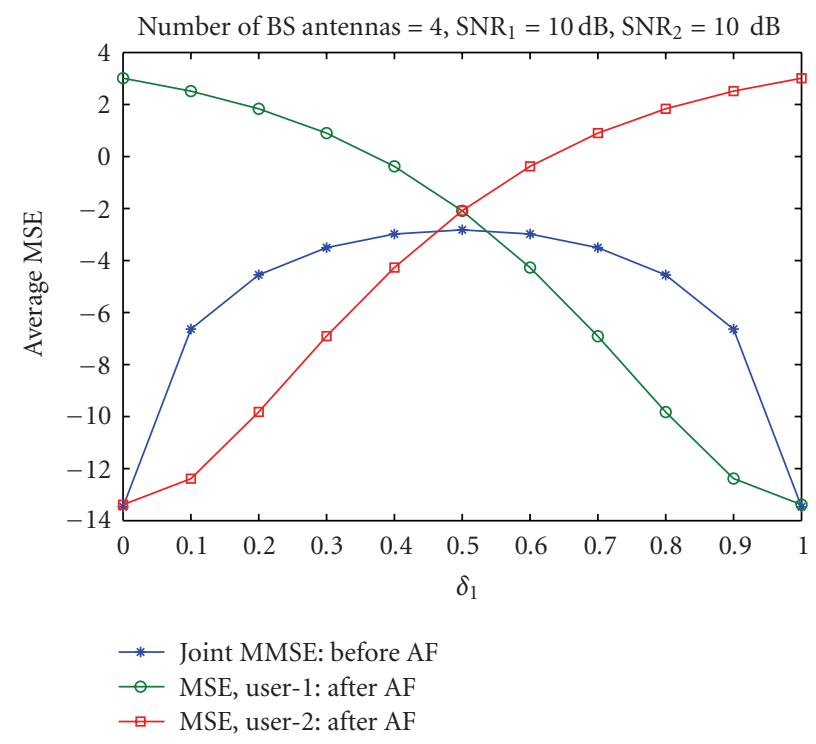

(a) Equal average uplink received SNRs

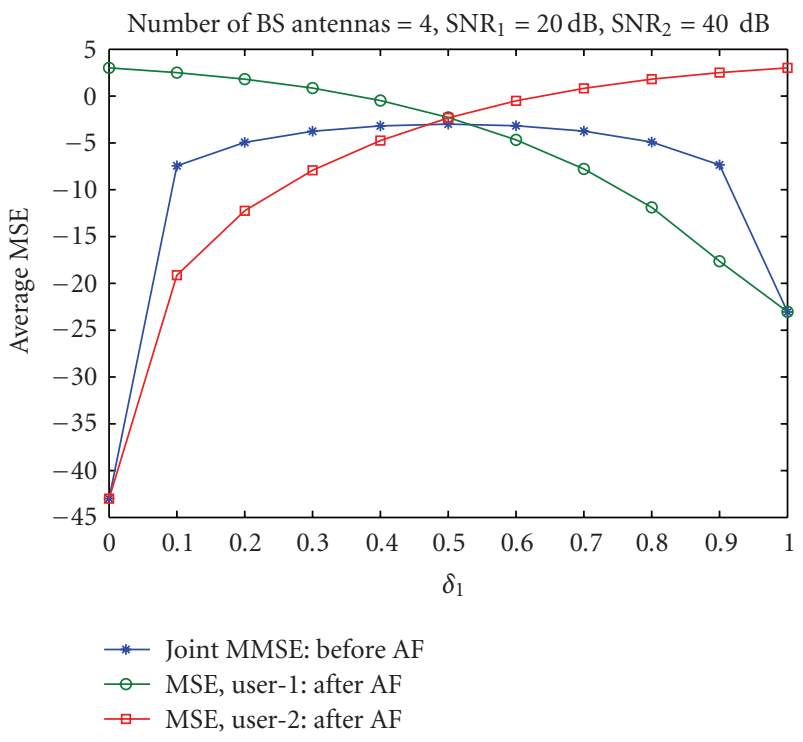

(b) Unequal average uplink received SNRs

FIgUre 4: Average MSE of MMSE-BAF system with 4 antennas at the base-station.

figures, (i) user-1 employs QPSK modulation whereas user2 employs 16-QAM modulation, (ii) a data frame contains 100 modulation symbols and 20 pilot symbols for channel estimation, and (iii) the channel remains constant over the duration of at least two frames (TDD assumption). In Figure 6, the base-station and the users all have single transmit/receive antennas. With equal average received SNRs at the base-station, Figure 6(a) shows that, at lower average received SNRs, pilot-based channel estimation matches very closely the performance achieved in case of perfect channel knowledge for both users. Since each user has to subtract its own channel-compensated transmitted symbol to decode the other user's modulation symbol, a user transmitting using a higher-order constellation has the potential to generate 


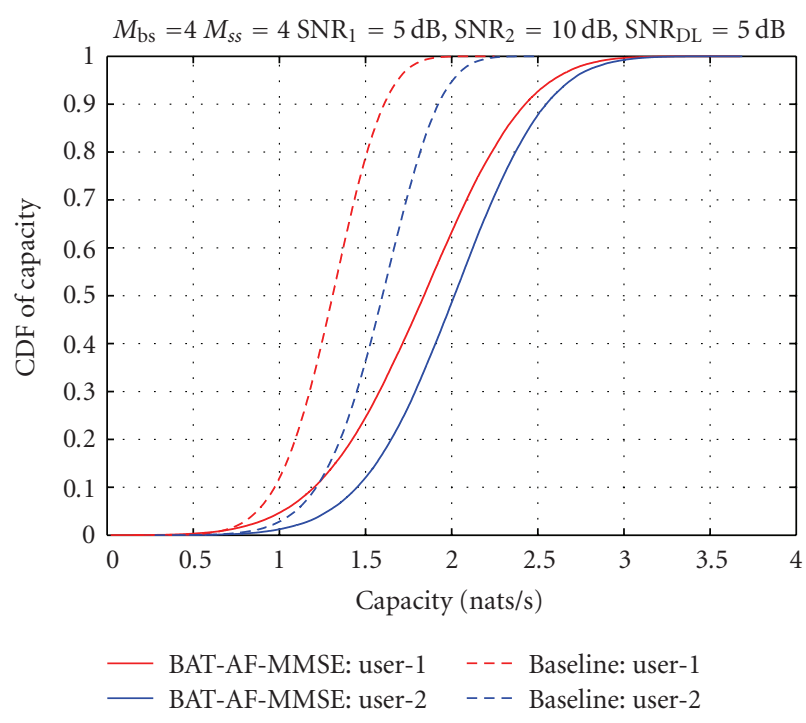

FIGURE 5: Comparison of empirical capacity CDFs of the MMSEBAF system against the baseline system with 4 antennas at the basestation as well as the mobile receivers.

higher self-interference in the presence of channel estimation errors. Figure 6(a) shows that with equal average received SNRs, the average SER of BPSK exhibits an error floor for an average SNR higher than $30 \mathrm{~dB}$. When user- 2 transmits at an SNR that is $20 \mathrm{~dB}$ higher than user-1's SNR, Figure 6(b) shows that the error floor for the BPSK modulation occurs much earlier.

The advantages of transmit antenna selection over transmitting from an arbitrary antenna is investigated in Figure 7 when the base-station has four antennas for reception. Exploiting the channel reciprocity of TDD systems, we first compute the elementwise magnitude of the estimated beamformer and downlink transmission is directed from the antenna that has the highest magnitude. It is important to note that once the base-station computes the MMSE beamformer, no additional computation complexity for TAS is required. From Figure 7, we observe that the pilot-based channel estimation has excellent performance in comparison with the ideal performance and our proposed simple TAS yields an impressive gain of close to $3 \mathrm{~dB}$ at an average SER of $10^{-4}$

We have also investigated the feasibility of bidirectional relaying for OFDM/OFDMA-based 4G cellular standards such as IEEE 802.16e [13]. The IEEE 802.16e system is based on OFDMA physical layer for both uplink and downlink. Current mobile WiMax standard supports various subchannelization procedures, in both uplink and downlink directions, for data transmission in time (OFDM symbols) and frequency (OFDM subcarriers). One such uplink subchannelization procedure is termed partially utilized subchannelization (PUSC) wherein the modulation symbols of a given user are pseudorandomly spread over the frequency band to extract frequency diversity and to average interference across neighboring cells/sectors. Briefly, one slot in UL-PUSC is defined as 48 modulation symbols

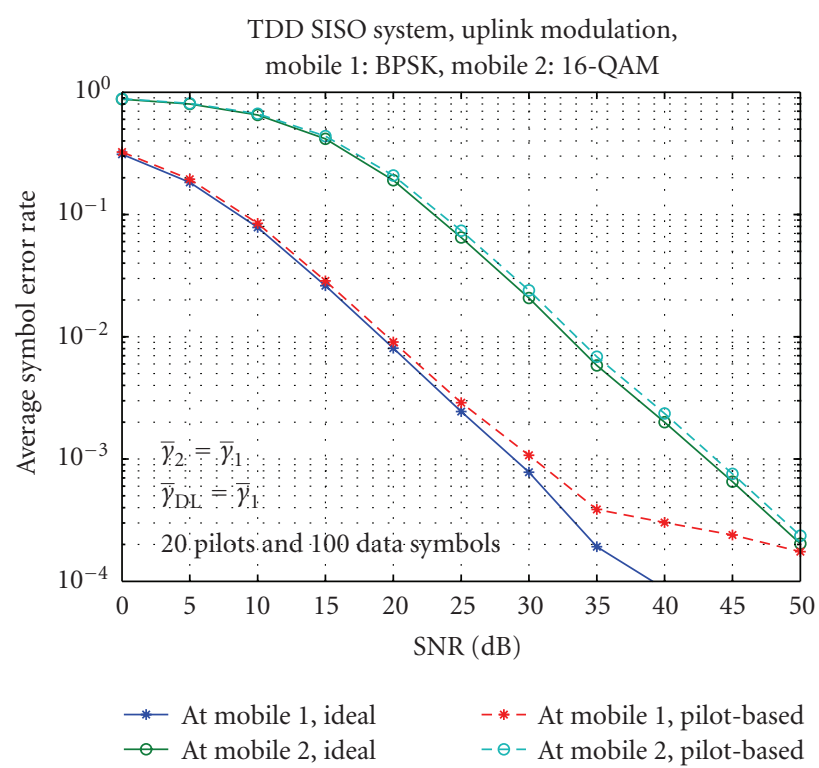

(a) Equal average uplink received SNRs

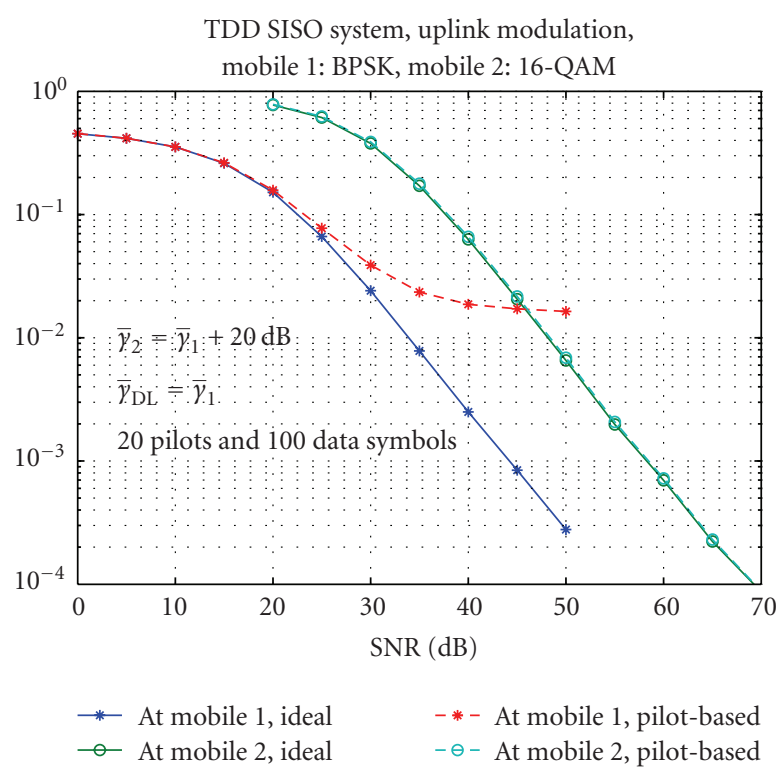

(b) Unequal average uplink received SNRs

FIGURE 6: Average uncoded SER of MMSE-BAF system with QPSK modulation for user 1 and 16-QAM modulation for user 2.

spanning over three consecutive OFDM symbols (which is a PUSC slot duration). The modulation symbols together with the pilot symbols needed to estimate the uplink channel are sent over 6 tiles distributed over frequency, where a tile is defined as four consecutive subcarriers over three consecutive OFDM symbols. Each tile contains 4 pilot symbols, placed at the corners of the tile, and 8 data symbols. An FEC block in WiMax comprises of a given number of slots and the maximum FEC block size is a function of the modulation order and channel coding rate. The WiMax standard supports 8 modulation order and coding rate combinations. These are QPSK modulation with 


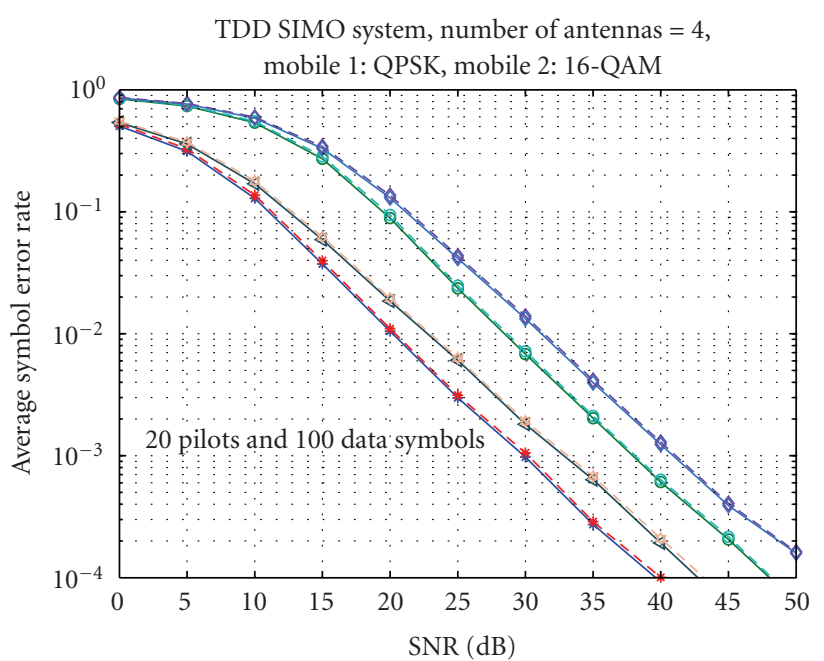

$$
\begin{aligned}
& \rightarrow-\text { At mobile 1, ideal, TAS } \\
& -\bullet \text { At mobile 2, ideal, TAS } \\
& -*-\text { At mobile 1, pilot-based, TAS } \\
& -\ominus-\text { At mobile 2, pilot-based, TAS } \\
& \triangleleft-\text { At mobile 1, ideal, no TAS } \\
& \diamond-\text { At mobile 2, ideal, no TAS } \\
& -- \text { At mobile 1, pilot-based, no TAS } \\
& -\diamond-\text { At mobile 2, pilot-based, no TAS }
\end{aligned}
$$

Figure 7: Performance of transmit antenna selection for TDDbased MMSE-BAF system with 4 antennas at the base-station. User1 employs QPSK modulation whereas user-2 employs 16-QAM modulation. An uncoded system is considered with realistic channel estimation over block fading channels with 20 pilot and 100 data symbols per fading block.

code rates $1 / 2$ and 3/4, 16-QAM modulation with code rates $1 / 2$ and $3 / 4$, and 64-QAM modulation with rates $1 / 2,2 / 3,3 / 4$, and $5 / 6$. Figure 8 shows the modified ULPUSC structure to support bidirectional communications. Each user employs Hadamard sequences as pilot symbols to enable the base-station to estimate the individual channels without interference. For downlink transmission, the basestation can use any subchannelization procedure. However, to render our proposal valid for FDD as well as TDD systems, the downlink subchannel structure is set identical to the uplink one and the broadcast pilots from each tile are used for channel estimation at the mobile stations. For efficient cancellation of self-interference, each mobile station requires the knowledge of uplink channel-related parameters $\beta$ (a positive scalar value) and the two complex scalars values $v_{1}:=\mathbf{w}_{\mathrm{opt}}^{\mathscr{H}} \mathbf{h}_{1}$ and $v_{2}:=\mathbf{w}_{\mathrm{opt}}^{\mathcal{H}} \mathbf{h}_{2}$. Additional simulation parameters are listed in Table 1.

In Figures 9 and 10, we show the performance of MMSEBAF when applied to an IEEE 802.16e system in an FDD mode of operation. These simulations are conducted for a base-station with four receive antennas and for a twoantenna mobile station receiver. In these plots, block error rate (BLER) performances of genie-aided perfect channel knowledge are compared against pilot-based realistic channel estimation schemes. For each tile, one channel estimate is obtained by sample averaging the received pilots over
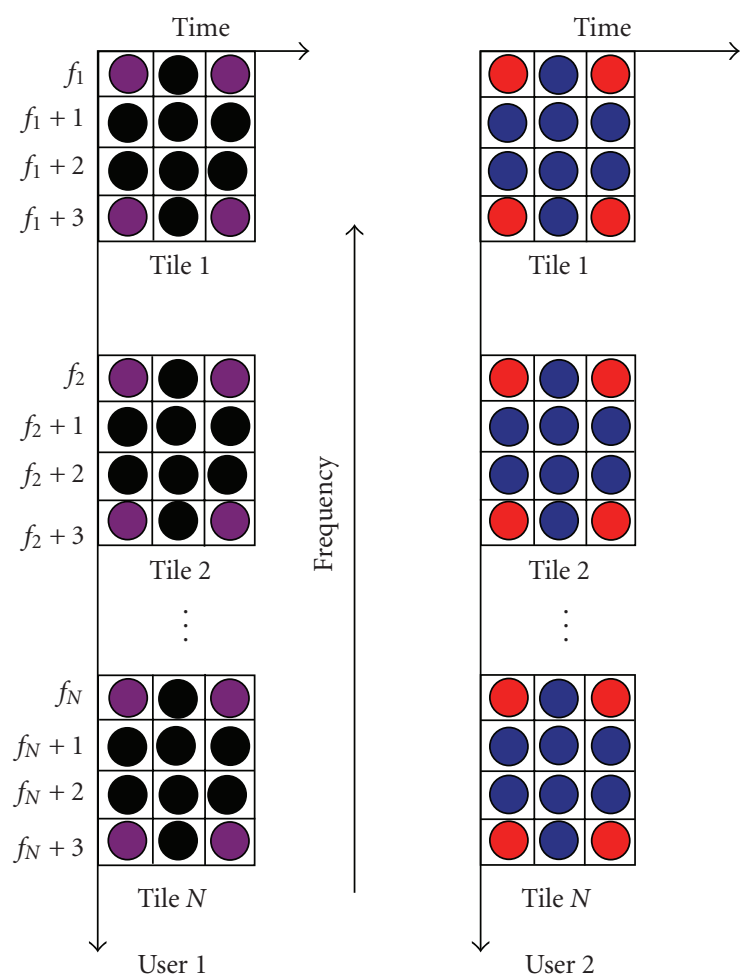

FIGURE 8: Subchannelization procedure for bidirectional communication using the IEEE 802.16e protocol. In uplink, each user is allocated $K$ slots spanning $N_{f}$ subchannels in frequency over $N_{t}$ slot-durations. Each slot-duration comprises 3 consecutive OFDM symbols, whereas each subchannel contains 6 tiles distributed throughout of the useful frequency band. A tile contains 4 subcarries over a slot-duration with the 4 pilots at the corners of the tile and the remaining 8 tones used for data. In the above figure, there are $N$ tiles with $N_{t}=1, N_{f}=N / 6$, and $K=N_{t} N_{f}=N / 6$. The circles filled with violet and red colors indicate the pilot tones of users 1 and 2, respectively, whereas the circles filled with black and blue colors indicate the data tones of users 1 and 2, respectively.

TABLE 1: Simulation parameters.

\begin{tabular}{lc}
\hline Parameter & Value \\
\hline Bandwidth & $10 \mathrm{MHz}$ \\
Sampling rate & $11.2 \mathrm{Msps}$ \\
FFT size & 1024 \\
Subcarrier spacing & $10.9375 \mathrm{KHz}$ \\
Useful symbol duration $\left(T_{u}\right)$ & $91.4286 \mu \mathrm{sec}$ \\
Cyclic prefix $\left(T_{G}\right)$ & $T_{u} / 8$ \\
Useful subcarriers & 840 \\
Left guard subcarriers & 92 \\
Right guard subcarriers & 91 \\
Channel coding & Convolutional turbo coding \\
& (with 8 iterations) \\
Carrier frequency & $2.0 \mathrm{GHz}$ \\
\hline
\end{tabular}

that tile. Knowledge of neither the fading statistics nor the delay/Doppler spread is assumed at the receivers. In Figure 9, both users are assumed to encode their data using 


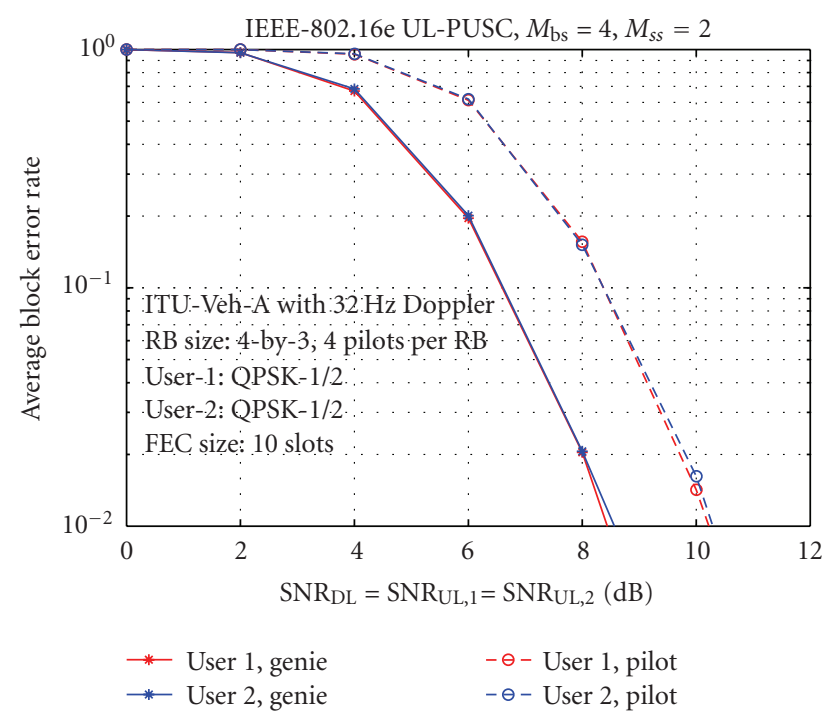

(a) With $32 \mathrm{~Hz}$ Doppler

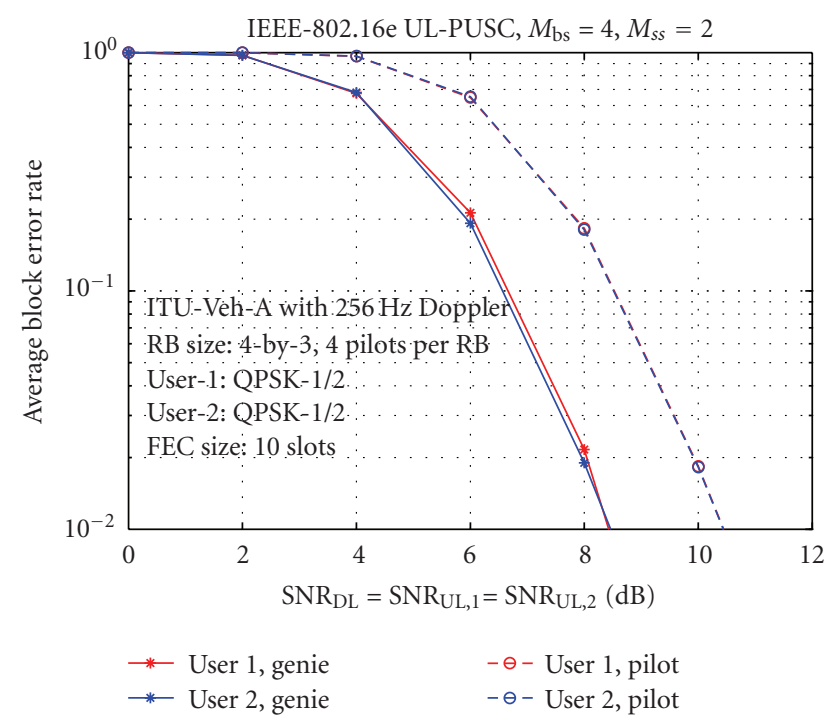

(b) With $256 \mathrm{~Hz}$ Doppler

FIgure 9: Performance of MMSE-BAF on MIMO-OFDMA-based IEEE 802.16e system using uplink partial utilization of subchannelization (PUSC) permutation. Each user employs rate-1/2 convolutional turbo coding (CTC) with QPSK modulation. The FEC block length is 480 information bits which corresponds to 10 slots, with 48 modulation symbols per slot, as per the terminology in [13].

QPSK modulation with rate-1/2 convolutional turbo coding (CTC). For this MCS, the FEC block size is set to the maximum allowed, which is equal to 10 slots or $10 \times 48 \times$ $2 \times 1 / 2=480$ information symbols. We have considered ITU Vehicular-A channel model with both low and high Doppler spread values of $32 \mathrm{~Hz}$ and $256 \mathrm{~Hz}$, respectively. Figure 9 shows that, under both high and low Doppler scenarios, pilot channel estimation incurs a loss of about $2 \mathrm{~dB}$, and MMSE-BAF works extremely well in supporting data exchanges in high-mobile environments. Figure 10 shows the

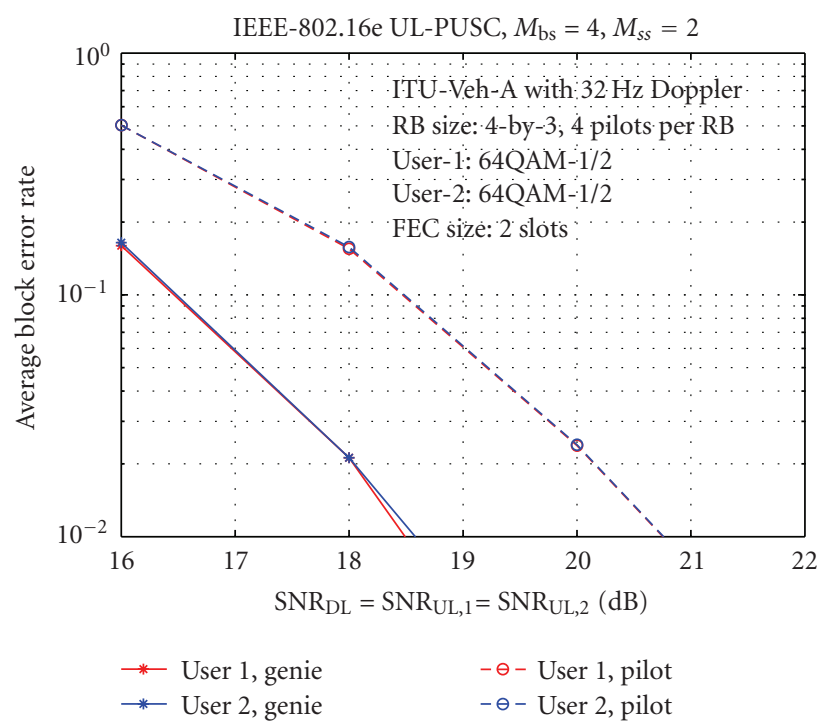

(a) ITU-Veh-A channel with $32 \mathrm{~Hz}$ Doppler

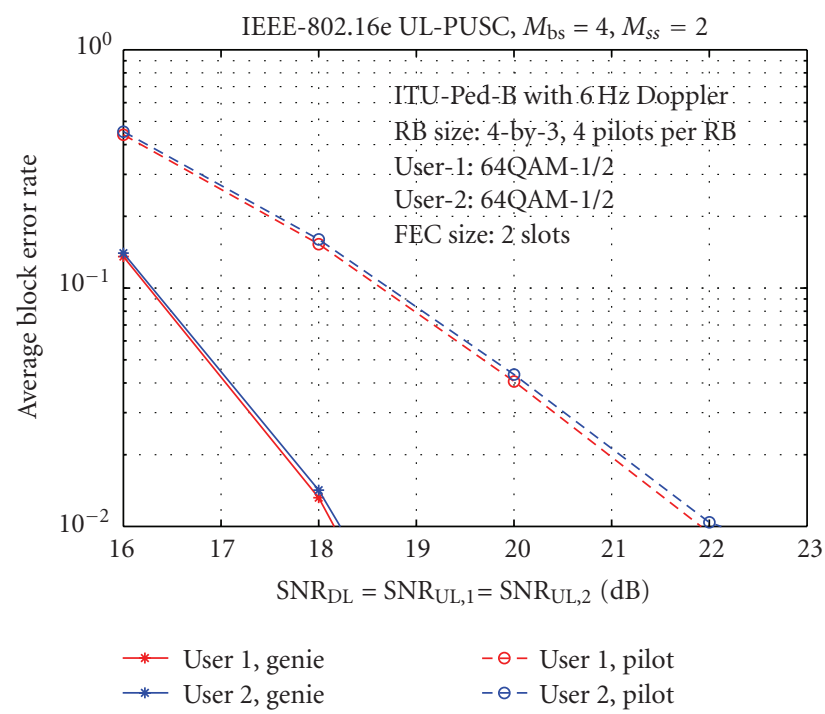

(b) ITU-Ped-B channel with $6 \mathrm{~Hz}$ Doppler

Figure 10: Performance of MMSE-BAF on MIMO-OFDMAbased IEEE 802.16e system using uplink partial utilization of subchannelization (PUSC) permutation. Each user employs rate$1 / 2$ convolutional turbo coding (CTC) with 64QAM modulation. The FEC block length is 288 information bits which corresponds to 2 slots, with 48 modulation symbols per slot, as per the terminology in [13].

performance of MMSE-BAF with 64-QAM modulation and a CTC with code rate of $1 / 2$. With this MCS level, supporting a per-user over-the-air spectral efficiency of $3 \mathrm{bits} / \mathrm{sec} / \mathrm{Hz}$, we have employed the maximum possible FEC block size of 2 slots, or $2 \times 48 \times 6 \times 1 / 2=288$ information bits. Figure 10 (a) shows the block error performance over ITU-Vehicular-A channel with $32 \mathrm{~Hz}$ Doppler, whereas Figure 10(b) shows the performance on a Pedestrian-B channel with $6 \mathrm{~Hz}$ Doppler. Due to higher-frequency-selectivity of Pedestrian-B channel, compared with the Vehicular-A channel where the 
channel estimation-based BLER performance (at 1 percent BLER) is about $2 \mathrm{~dB}$ away from the ideal performance, the simple sample-average-based channel estimation has a performance degradation of about $3.5 \mathrm{~dB}$ compared to the performance with perfect channel knowledge. It is expected that the BLER performance can be significantly improved by incorporating a more complex two-dimensional channel estimation scheme, such as Wiener filtering, which requires knowledge of fading statistics as well as delay and Doppler spread information.

\section{Conclusion}

In this paper, we have introduced a so-called MMSE-BAF protocol for bidirectional communications over two-way relay channels with multiantenna relay nodes. The features of this protocol include the usage of analog network coding at the relay node and the evaluation of a receive weight vector for the relay node using a joint linear MMSE filtering operation on the received uplink multiple access signal. Transmit antenna selection using the largest MMSE weight branch on the downlink is also an inherent feature of the proposed protocol in the TDD mode of operation. Extensive link-level simulations have been proposed for both TDD and FDD modes of operations and required modifications to the existing IEEE 802.16e standard have been proposed to accommodate the MMSE-BAF protocol. It has been shown through simulation results that the MMSEBAF protocol is a simple yet efficient solution to the problem of bidirectional communications in two-way relay channels with multiantenna relays and half-duplex nodes.

\section{Appendix}

\section{Received SNRs with Noise Whitening}

We first rewrite the noise covariance matrices $\boldsymbol{\Sigma}_{1}(10)$ and $\boldsymbol{\Sigma}_{2}$ (20), using their eigenvalue decompositions, as

$$
\begin{aligned}
& \boldsymbol{\Sigma}_{1}=\mathbf{P}_{1}^{\mathcal{H}} \boldsymbol{\Lambda}_{1} \mathbf{P}_{1}, \\
& \boldsymbol{\Sigma}_{2}=\mathbf{P}_{2}^{\mathcal{H}} \boldsymbol{\Lambda}_{2} \mathbf{P}_{2},
\end{aligned}
$$

where $\mathbf{P}_{1}$ and $\mathbf{P}_{2}$ are unitary matrices and $\boldsymbol{\Lambda}_{1}$ and $\boldsymbol{\Lambda}_{2}$ are diagonal matrices containing the eigenvalues of $\boldsymbol{\Sigma}_{1}$ and $\boldsymbol{\Sigma}_{2}$, respectively.

Let us now focus on demodulating $x_{2}$ from $\mathbf{r}_{1}$ by whitening the noise $\tilde{\mathbf{n}}_{1}$. Let

$$
\begin{aligned}
\mathbf{s}_{1} & =\boldsymbol{\Lambda}_{1}^{-1 / 2} \mathbf{P}_{1} \mathbf{r}_{1} \\
& =\Lambda_{1}^{-1 / 2} \mathbf{P}_{1}\left(\mathbf{g}_{1} \beta v_{2} x_{2}+\widetilde{\mathbf{n}}_{1}\right) .
\end{aligned}
$$

Since, conditioned on $\boldsymbol{\Lambda}_{1}$ and $\mathbf{P}$,

$$
\mathrm{E}\left[\left(\boldsymbol{\Lambda}_{1}^{-1 / 2} \mathbf{P}_{1} \widetilde{\mathbf{n}}_{1}\right)\left(\boldsymbol{\Lambda}_{1}^{-1 / 2} \mathbf{P}_{1} \widetilde{\mathbf{n}}_{1}\right)^{\mathscr{H}}\right]=\mathbf{I}_{M_{s s}},
$$

it follows from (A.2) that the instantaneous received SNR of $x_{2}$ by whitening $\mathbf{r}_{1}$ is simply

$$
\begin{aligned}
\gamma_{2}^{\prime} & =\sigma_{2}^{2}\left\|\boldsymbol{\Lambda}_{1}^{-1 / 2} \mathbf{P}_{1} \mathbf{g}_{1} \beta v_{2}\right\|^{2} \\
& =\sigma_{2}^{2} \beta^{2}\left|v_{2}\right|^{2} \mathbf{g}_{1}^{\mathscr{H}} \mathbf{P}_{1}^{\mathcal{H}} \boldsymbol{\Lambda}_{1}^{-1 / 2} \boldsymbol{\Lambda}_{1}^{-1 / 2} \mathbf{P}_{1} \mathbf{g}_{1} \\
& =\sigma_{2}^{2} \beta^{2}\left|v_{2}\right|^{2} \mathbf{g}_{1}^{\mathcal{H}} \mathbf{P}_{1}^{\mathcal{H}} \boldsymbol{\Sigma}_{1}^{-1} \mathbf{g}_{1} .
\end{aligned}
$$

In a similar manner, upon whitening $\mathbf{r}_{2}$ to demodulate $x_{1}$, the instantaneous received SNR of $x_{1}$ becomes

$$
\gamma_{1}^{\prime}=\sigma_{1}^{2} \beta^{2}\left|\nu_{1}\right|^{2} \mathbf{g}_{2}^{\mathcal{H}} \boldsymbol{\Sigma}_{2}^{-1} \mathbf{g}_{2}
$$

Using the following matrix inversion lemma (MIL) [14]

$$
\left(\mathbf{I}_{M_{s s}}+\mathbf{x} \mathbf{x}^{\mathscr{H}}\right)^{-1}=\mathbf{I}_{M_{s s}}-\frac{\mathbf{x x}^{\mathscr{H}}}{1+\|\mathbf{x}\|^{2}},
$$

where $\mathbf{x}$ is a column-vector of appropriate size, it is possible to further simplify (A.4) as

$$
\begin{aligned}
\gamma_{2}^{\prime} & =\frac{\sigma_{2}^{2} \beta^{2}\left|v_{2}\right|^{2}}{\sigma_{N}^{2}} \times \mathbf{g}_{1}^{\mathscr{H}}\left(\mathbf{I}_{M_{s s}}-\frac{\beta^{2}\left\|\mathbf{w}_{\mathrm{opt}}\right\|^{2} \mathbf{g}_{1} \mathbf{g}_{1}^{\mathcal{H}}}{1+\beta^{2}\left\|\mathbf{w}_{\mathrm{opt}}\right\|^{2}\left\|\mathbf{g}_{1}\right\|^{2}}\right) \mathbf{g}_{1} \\
& =\frac{\sigma_{2}^{2} \beta^{2}\left|v_{2}\right|^{2}}{\sigma_{N}^{2}} \times \frac{\left\|\mathbf{g}_{1}\right\|^{2}}{1+\beta^{2}\left\|\mathbf{w}_{\mathrm{opt}}\right\|^{2}\left\|\mathbf{g}_{1}\right\|^{2}} .
\end{aligned}
$$

In a similar manner, application of MIL in (A.5) leads to

$$
\gamma_{1}^{\prime}=\frac{\sigma_{1}^{2} \beta^{2}\left|v_{1}\right|^{2}}{\sigma_{N}^{2}} \times \frac{\left\|\mathbf{g}_{2}\right\|^{2}}{1+\beta^{2}\left\|\mathbf{w}_{\mathrm{opt}}\right\|^{2}\left\|\mathbf{g}_{2}\right\|^{2}}
$$

As we mentioned earlier, expressions for $\gamma_{2}^{\prime}$ (A.7) and $\gamma_{1}^{\prime}$ (A.8) are, respectively, identical to SNRs $\gamma_{2}(21)$ and $\gamma_{1}(18)$ derived in Section 3.2 without performing noise whitening. This somewhat counterintuitive result leads us to conclude that SNR improvement is not an option with noise whitening when employing the MMSE-BAF protocol.

\section{References}

[1] Y. Wu, P. A. Chou, and S.-Y. Kung, "Information exchange in wireless networks with network coding and physical-layer broadcast," in Proceedings of the 39th Annual Conference on information Sciences and Systems (CISS '05), March 2005.

[2] S. Katti, S. Gollakota, and D. Katabi, "Embracing wireless interference: analog netwok coding," Tech. Rep. MIT-CSAILTR-2007-012, Computer Science and Artificial Intelligence Laboratory, Cambridge, Mass, USA, February 2007.

[3] S. Zhang, S. C. Liew, and P. P. Lam, "Hot topic: physicallayer network coding," in Proceedings of the 12th Annual International Conference on Mobile Computing and Networking (MOBICOM '06), pp. 358-365, Los Angeles, Calif, USA, September 2006.

[4] S. J. Kim, P. Mitran, and V. Tarokh, "Performance bounds for bidirectional coded cooperation protocols," IEEE Transactions on Information Theory, vol. 54, no. 11, pp. 5235-5241, 2008. 
[5] S. J. Kim, N. Devroye, P. Mitran, and V. Tarokh, "Achievable rate regions for bi-directional relaying," http://www.citebase .org/abstract?id=oai:arXiv.org:0808.0954.

[6] S. J. Kim, N. Devroye, and V. Tarokh, "Bi-directional halfduplex protocols with multiple relays," http://www.citebase .org/abstract?id=oai:arXiv.org:0810.1268.

[7] P. Popovski and H. Yomo, "The anti-packets can increase the achievable throughput of a wireless multi-hop network," in Proceedings of the IEEE International Conference on Communications (ICC '06), vol. 9, pp. 3885-3890, Istanbul, Turkey, June 2006.

[8] P. Popovski and H. Yomo, "Wireless network coding by amplify-and-forward for bi-directional traffic flows," IEEE Communications Letters, vol. 11, no. 1, pp. 16-18, 2007.

[9] P. Popovski and H. Yomo, "Physical network coding in two-way wireless relay channels," in Proceedings of the IEEE International Conference on Communications (ICC '07), pp. 707-712, Glasgow, Scotland, June 2007.

[10] I. Hammerström, M. Kuhn, C. Eşli, J. Zhao, A. Wittneben, and G. Bauch, "MIMO two-way relaying with transmit CSI at the relay," in Proceedings of the 8th IEEE Workshop on Signal Processing Advances in Wireless Communications (SPAWC '07), pp. 1-5, Helsinki, Finland, June 2007.

[11] T. Unger and A. Klein, "Duplex schemes in multiple antenna two-hop relaying," EURASIP Journal on Advances in Signal Processing, vol. 2008, Article ID 128592, 14 pages, 2008.

[12] R. Zhang, Y.-C. Liang, C. C. Chai, and S. Cui, "Optimal beamforming for two-way multi-antenna relay channel with analogue network coding," IEEE Journal on Selected Areas in Communications, vol. 27, no. 5, pp. 699-712, 2009.

[13] "IEEE Standard for Local and Metropolitan Area Networks, Amendment 2: Physical and Medium Access Control Layers for Combined Fixed and Mobile Operation in Licensed Bands," IEEE Std. 802.16-2005, 2005.

[14] G. H. Golub and C. F. V. Loan, Matrix Computations, The Johns Hopkins University Press, Baltimore, Md, USA, 3rd edition, 1996. 

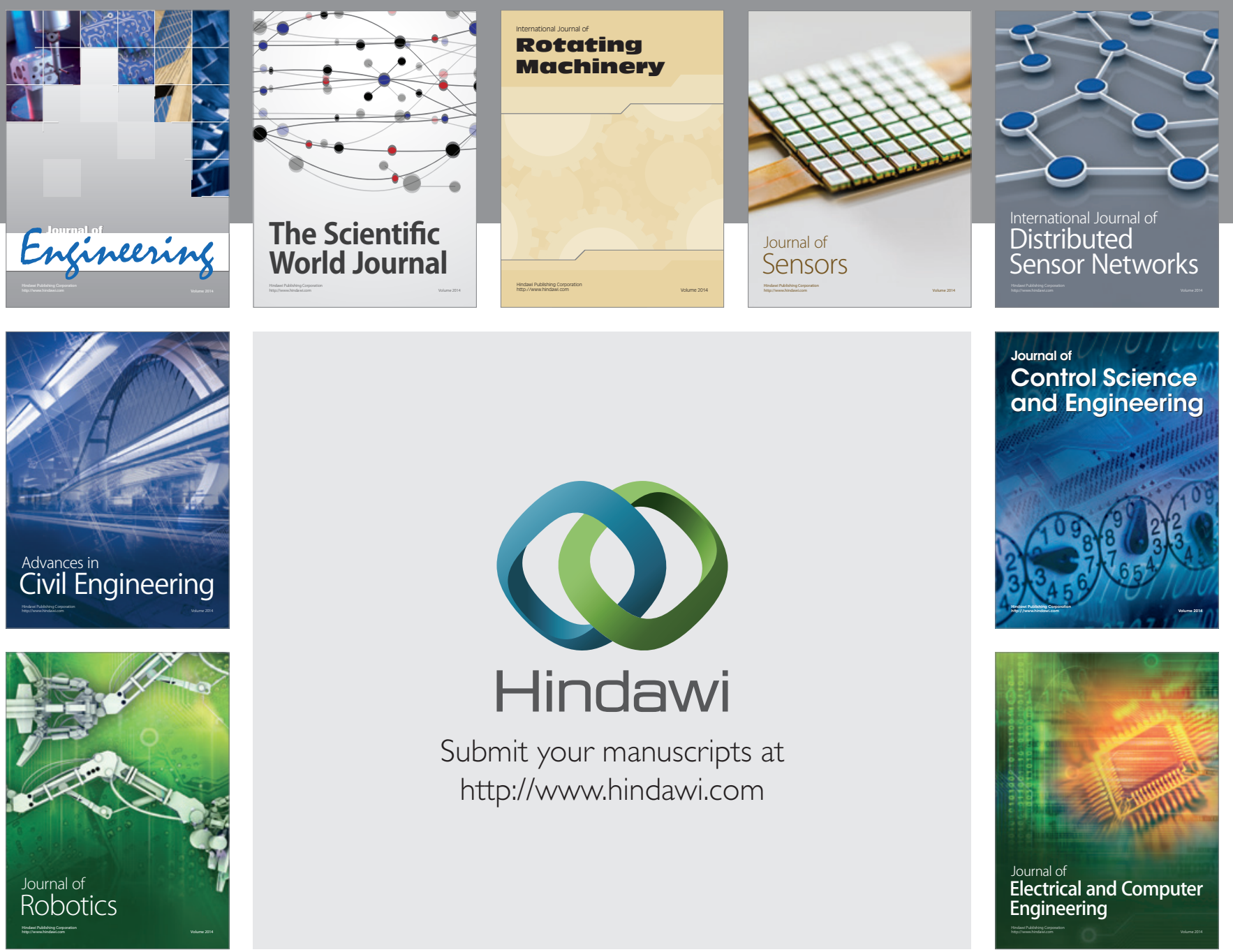

Submit your manuscripts at

http://www.hindawi.com
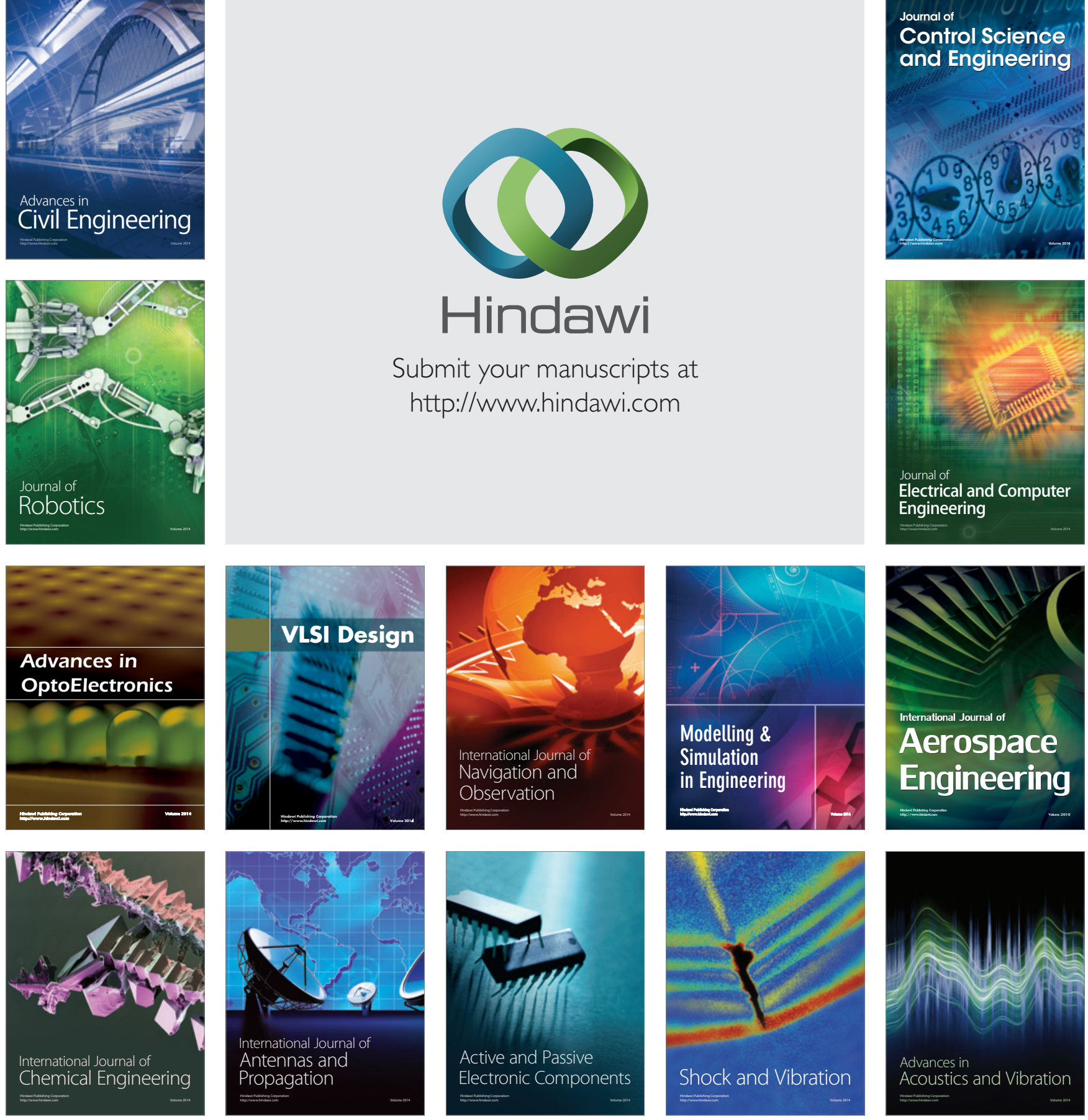